\title{
Deterministic Graph Exploration with Advice
}

\author{
Barun Gorain ${ }^{1}$ and Andrzej Pelc*2 \\ 1 Département d'informatique, Université du Québec en Outaouais, Gatineau, \\ Québec, Canada \\ baruniitg123@gmail.com \\ 2 Département d'informatique, Université du Québec en Outaouais, Gatineau, \\ Québec, Canada \\ pelc@uqo.ca
}

\begin{abstract}
We consider the task of graph exploration. An $n$-node graph has unlabeled nodes, and all ports at any node of degree $d$ are arbitrarily numbered $0, \ldots, d-1$. A mobile agent has to visit all nodes and stop. The exploration time is the number of edge traversals. We consider the problem of how much knowledge the agent has to have a priori, in order to explore the graph in a given time, using a deterministic algorithm. This a priori information (advice) is provided to the agent by an oracle, in the form of a binary string, whose length is called the size of advice. We consider two types of oracles. The instance oracle knows the entire instance of the exploration problem, i.e., the port-numbered map of the graph and the starting node of the agent in this map. The map oracle knows the port-numbered map of the graph but does not know the starting node of the agent. What is the minimum size of advice that must be given to the agent by each of these oracles, so that the agent explores the graph in a given time?

We first consider exploration in polynomial time, and determine the exact minimum size of advice to achieve it. This size is $\log \log \log n-\Theta(1)$, for both types of oracles.

When advice is large, there are two natural time thresholds: $\Theta\left(n^{2}\right)$ for a map oracle, and $\Theta(n)$ for an instance oracle, that can be achieved with sufficiently large advice. We show that, with a map oracle, time $\Theta\left(n^{2}\right)$ cannot be improved in general, regardless of the size of advice. We also show that the smallest size of advice to achieve this time is larger than $n^{\delta}$, for any $\delta<1 / 3$.

For an instance oracle, advice of size $O(n \log n)$ is enough to achieve time $O(n)$. We show that, with any advice of size $o(n \log n)$, the time of exploration must be at least $n^{\epsilon}$, for any $\epsilon<2$, and with any advice of size $O(n)$, the time must be $\Omega\left(n^{2}\right)$.

We also investigate minimum advice sufficient for fast exploration of Hamiltonian graphs.
\end{abstract}

1998 ACM Subject Classification F.2.2 Nonnumerical Algorithms and Problems

Keywords and phrases algorithm, graph, exploration, mobile agent, advice

Digital Object Identifier 10.4230/LIPIcs.ICALP.2017.132

\section{Introduction}

Exploration of networks by visiting all of their nodes is one of the basic tasks performed by a mobile agent in networks. In applications, a software agent may need to collect data placed at nodes of a network, or a mobile robot may need to collect samples of air or ground in a contaminated mine whose corridors form links of a network, with corridor crossings represented by nodes.

\footnotetext{
* Research supported in part by NSERC Discovery Grant 8136 - 2013 and by the Research Chair in
} Distributed Computing of the Université du Québec en Outaouais. 
The network is modeled as a simple connected undirected graph $G=(V, E)$ with $n$ nodes, called graph in the sequel. Nodes are unlabeled, and all ports at any node of degree $d$ are arbitrarily numbered $0, \ldots, d-1$. The agent is initially situated at a starting node $v$ of the graph. When the agent located at a current node $u$ gets to a neighbor $w$ of $u$ by taking port $i$, it learns the port $j$ by which it enters node $w$ and it learns the degree of $w$. The agent has to visit all nodes of the graph and stop. The agent is computationally unbounded and cannot mark the visited nodes.

The time of the exploration is the number of edge traversals. We consider the problem of how much knowledge the agent has to have a priori, in order to explore the graph in a given time, using a deterministic algorithm. It is well-known that some information is necessary, as witnessed even by the class of rings in which ports at all nodes are numbered 0,1 in clockwise order. Navigating in such a ring, the agent cannot learn its size. If there existed an exploration algorithm not using any a priori knowledge, then it would have to stop after some $t$ steps in every ring, and hence would fail to explore a $(t+2)$-node ring.

Following the paradigm of algorithms with advice $[1,13,17,18,21,20,22,23,24,25,26$, $27,29]$, this a priori information (advice), needed for exploration, is provided to the agent by an oracle, in the form of a binary string, whose length is called the size of advice. We consider two types of oracles. An instance oracle knows the entire instance of the exploration problem, i.e., the port-numbered map of the graph and the starting node of the agent in this map. A map oracle knows the port-numbered map of the graph but does not know the starting node of the agent. Formally, a map oracle is a function $f: \mathcal{G} \longrightarrow S$, where $\mathcal{G}$ is the set of graphs and $S$ is the set of finite binary strings. An instance oracle is a function $f: \mathcal{I} \longrightarrow S$, where $\mathcal{I}$ is the set of pairs $(G, v)$, with $G \in \mathcal{G}$ and $v$ being the starting node of the agent in graph $G$. The advice $s$ is an input to an exploration algorithm. We say that exploration in time $t$ with advice of size $x$ given by an instance oracle is possible, if there exists advice of length $x$ depending on the instance $(G, v)$, and an exploration algorithm using this advice, which explores every graph in time $t$, starting from node $v$. Likewise, we say that exploration in time $t$ with advice of size $x$ given by a map oracle is possible, if there exists advice of length $x$ depending on the graph $G$, and an exploration algorithm using this advice, which explores every graph in time $t$, starting from any node. (Integers $x$ and $t$ depend on the size of the graph.) Proving that such an exploration is possible consists in showing an oracle of the appropriate type giving advice of size $x$, and an exploration algorithm using this advice and working in time $t$, for any graph and any starting node. Proving that such an exploration is impossible consists in showing that, for any oracle of the appropriate type giving advice of size $x$, and for any exploration algorithm using it, there exists a graph and a starting node for which this algorithm will exceed time $t$.

The central question studied in this paper is:

What is the minimum size of advice that has to be given to the agent by an instance oracle (resp. by a map oracle) to permit the agent to explore any graph in a given time?

Our main contributions are negative results of two types:

- impossibility results showing that the less powerful map oracle cannot help to achieve the same exploration time as the more powerful instance oracle, regardless of the size of advice;

- lower bounds showing that the size of some natural advice leading to a simple exploration in a given time cannot be improved significantly.

While in most cases our bounds on the size of advice are asymptotically tight, in one case the remaining gap is cubic. 


\subsection{Our results}

We first consider exploration in polynomial time, and determine the exact minimum size of advice to achieve it. Indeed, we prove that some advice of size $\log \log \log n-c$, for any constant $c$, is sufficient to permit polynomial exploration of all $n$-node graphs, and that no advice of size $\log \log \log n-\phi(n)$, where $\phi$ is any function diverging to infinity, can help to do this. Both these results hold both for the instance and for the map oracles.

On the other side of the spectrum, when advice is large, there are two natural time thresholds: $\Theta\left(n^{2}\right)$ for a map oracle, and $\Theta(n)$ for an instance oracle. This is because, in both cases, these time benchmarks can be achieved with sufficiently large advice (advice of size $O(n \log n)$ suffices). We show that, with a map oracle, time $\Theta\left(n^{2}\right)$ cannot be improved in general, regardless of the size of advice. What is then the smallest advice to achieve time $\Theta\left(n^{2}\right)$ with a map oracle? We show that this smallest size of advice is larger than $n^{\delta}$, for any $\delta<1 / 3$.

For large advice, the situation changes significantly when we allow an instance oracle instead of a map oracle. In this case, advice of size $O(n \log n)$ is enough to achieve time $O(n)$. Is such a large advice needed to achieve linear time? We answer this question affirmatively. Indeed, we show more: with any advice of size $o(n \log n)$, the time of exploration must be at least $n^{\epsilon}$, for any $\epsilon<2$, and with any advice of size $O(n)$, the time must be $\Omega\left(n^{2}\right)$.

We finally look at Hamiltonian graphs, as for them it is possible to achieve the absolutely optimal exploration time $n-1$, when sufficiently large advice (of size $O(n \log n)$ ) is given by an instance oracle. We show that a map oracle cannot achieve this: regardless of the size of advice, the time of exploration must be $\Omega\left(n^{2}\right)$, for some Hamiltonian graphs. On the other hand, even for an instance oracle, with advice of size $o(n \log n)$, optimal time $n-1$ cannot be achieved: indeed, we show that the time of exploration with such advice must sometimes exceed the optimal time $n-1$ by a summand $n^{\epsilon}$, for any $\epsilon<1$.

Our results permit us to compare advice of different size and of different quality. The size is defined formally, and for quality we may say that advice given by an instance oracle is superior to advice given by a map oracle, because an instance oracle, seeing not only the graph but also the starting node of the agent, can use the allowed bits of advice in a better way. Looking from this perspective it turns out that both size and quality of advice provably matter. The fact that quality of advice matters is proved by the following pair of results: for a map oracle, time $\Theta\left(n^{2}\right)$ cannot be beaten, regardless of the size of advice, while for an instance oracle time $O(n)$ can be achieved with $O(n \log n)$ bits of advice. The fact that the size of advice matters (with the same quality) is proved by the following pair of results: for an instance oracle, time $O(n)$ can be achieved with $O(n \log n)$ bits of advice, but with $o(n \log n)$ bits of advice time must be at least $n^{\epsilon}$, for any $\epsilon<2$.

\subsection{Related work}

The problem of exploration and navigation of mobile agents in an unknown environment has been extensively studied in the literature for many decades (cf. the survey [33]). The explored environment has been modeled in two distinct ways: either as a geometric terrain in the plane, e.g., an unknown terrain with convex obstacles [9], or a room with polygonal [12] or rectangular [5] obstacles, or as we do in this paper, i.e., as a graph, assuming that the agent may only move along its edges. The graph model can be further specified in two different ways: either the graph is directed, in which case the agent can move only from tail to head of a directed edge $[2,6,7,13]$, or the graph is undirected (as we assume) and the agent can traverse edges in both directions $[4,8,16,30,31]$. The efficiency measure 
adopted in most papers dealing with exploration of graphs is the time (or cost) of completing this task, measured by the number of edge traversals by the agent. Some authors $[4,8,16]$ impose further restrictions on the moves of the agent.

Another direction of research concerns exploration of anonymous graphs. In this case it is impossible to explore arbitrary graphs and stop after exploration, if no marking of nodes is allowed, and if nothing is known about the graph. Hence some authors [6, 7] allow pebbles which the agent can drop on nodes to recognize already visited ones, and then remove them and drop them in other places. A more restrictive scenario assumes a stationary token that is fixed at the starting node of the agent [11, 32]. Exploring anonymous graphs without the possibility of marking nodes (and thus possibly without stopping) is investigated, e.g., in $[14,19]$. The authors concentrate attention not on the cost of exploration but on the minimum amount of memory sufficient to carry out this task. In the absence of marking nodes, in order to guarantee stopping after exploration, some knowledge about the graph is required, e.g., an upper bound on its size [11, 34].

Providing nodes or agents with arbitrary kinds of information that can be used to perform network tasks more efficiently has been previously proposed in $[1,13,17,18,21,20,22$, $23,24,25,26,27,29]$ in contexts ranging from graph coloring to broadcasting and leader election. This approach was referred to as algorithms with advice. The advice is given either to nodes of the network or to mobile agents performing some network task. In the first case, instead of advice, the term informative labeling schemes is sometimes used, if different nodes can get different information.

Several authors studied the minimum size of advice required to solve network problems in an efficient way. In [27], given a distributed representation of a solution for a problem, the authors investigated the number of bits of communication needed to verify the legality of the represented solution. In [21], the authors compared the minimum size of advice required to solve two information dissemination problems using a linear number of messages. In [22], it was shown that advice of constant size given to the nodes enables the distributed construction of a minimum spanning tree in logarithmic time. In [15, 17], the advice paradigm was used for online problems. In particular, in [15] the authors studied online graph exploration with advice in labeled weighted graphs. In the case of [29], the issue was not efficiency but feasibility: it was shown that $\Theta(n \log n)$ is the minimum size of advice required to perform monotone connected graph clearing. In [26], the authors studied radio networks for which it is possible to perform centralized broadcasting in constant time. In [24], the authors studied the problem of topology recognition with advice given to nodes. The topic of [28] and [35] was the size of advice needed for fast leader election, resp. in anonymous trees and in arbitrary anonymous graphs. Exploration with advice was previously studied only for trees [20], and algorithm performance was measured using the competitive approach. In the present paper, the performance measure of an algorithm is the order of magnitude of exploration time, and hence the case of trees is trivial, as they can be explored in linear time without any advice.

\section{Exploration in polynomial time}

As a warm-up, we first consider the following question: What is the minimum size of advice permitting the agent to explore any graph in time polynomial in the size of the graph? In this section we give the exact answer to this question, both for the instance oracle and for the map oracle.

It is well-known that, if the agent knows an upper bound $n^{\prime}$ on the number $n$ of nodes of the graph, then exploration in time polynomial in $n^{\prime}$ is possible, starting from any node of the 
graph. The first result implying this fact was proved in [3]. The exploration proposed there works in time $O\left(n^{\prime 5} \log n^{\prime}\right)$, and is based on Universal Traversal Sequences (UTS). Later on, an exploration algorithm working in time polynomial in $n^{\prime}$ based on Universal Exploration Sequences (UXS) was established in [34]. While the polynomial in the latter paper has much higher degree, the solution from [34] can be carried out in logarithmic memory. Both UTS and UXS permit to find a sequence of port numbers to be followed by the agent, regardless of the topology of the graph and of its starting node. In the case of UTS, the sequence of port numbers to be followed is the UTS itself, and in the case of UXS it is constructed term by term, on the basis of the UXS and of the port number by which the agent entered the current node. Regardless of which solution is used, we have the following proposition:

- Proposition $1([3,34])$. If the agent knows an upper bound $n^{\prime}$ on the number $n$ of nodes of the graph, there exists an algorithm with input $n^{\prime}$ that permits the agent starting at any node of the graph to explore the graph and stop after $P\left(n^{\prime}\right)$ steps, where $P$ is some polynomial.

The positive part of our result on minimum advice is formulated in the following lemma. Its proof is based on Proposition 1. The advice given to the agent is some prefix of the binary representation of the number $\lfloor\log \log n\rfloor$, on the basis of which the agent computes a rough but sufficiently precise upper bound on the size of the graph which permits it to explore the graph, in time polynomial in its size.

- Lemma 2. For any positive constant c, there exists an exploration algorithm using advice of size $\lfloor\log \log \log n-c\rfloor$, that works in time polynomial in $n$, for any $n$-node graph.

The next result shows that the upper bound from the previous lemma is tight. Indeed, the following lower bound holds even for oriented rings, i.e., rings in which ports 0 and 1 are in clockwise order at every node.

$\checkmark$ Lemma 3. For any function $\phi: \mathbb{N} \rightarrow \mathbb{N}$ such that $\phi(n) \rightarrow \infty$ as $n \rightarrow \infty$, it is not possible to explore an n-node oriented ring in polynomial time, using advice of size at most $\log \log \log n-\phi(n)$.

Notice that Lemmas 2 and 3 hold both for the instance oracle and for the map oracle. The positive result from Lemma 2 holds even for the map oracle, as the advice concerns the size of the graph and does not require knowing the starting node of the agent. The negative result from Lemma 3 holds even for the instance oracle, as it is true even in oriented rings, where knowledge of the starting node does not provide any insight, since all nodes look the same. Hence Lemmas 2 and 3 imply the following theorem that gives a precise answer to the question stated at the beginning of this section.

- Theorem 4. The minimum size of advice permitting the agent to explore any graph in time polynomial in the size $n$ of the graph is $\log \log \log n-\Theta(1)$, both for the instance oracle and for the map oracle.

\section{Fast exploration}

When advice given to the agent can be large, there are two natural time thresholds: $\Theta\left(n^{2}\right)$ for a map oracle, and $\Theta(n)$ for an instance oracle. This is because, in both cases, these time benchmarks can be achieved with sufficiently large advice. Indeed, we have the following proposition. 


\section{- Proposition 5.}

1. There exists an exploration algorithm, working in time $O\left(n^{2}\right)$ and using advice of size $O(n \log n)$, provided by a map oracle, for $n$-node graphs.

2. There exists an exploration algorithm, working in time $O(n)$ and using advice of size $O(n \log n)$, provided by an instance oracle, for $n$-node graphs.

In the rest of this section we prove negative results indicating the quality of the natural solution given in Proposition 5. For the map oracle, we show that quadratic exploration time cannot be beaten, and we give a lower bound on the size of advice sufficient to guarantee this time. For the instance oracle, we show that Proposition 5 gives optimal advice for linear exploration time.

\subsection{Map oracle}

Our first result for the map oracle shows that, regardless of the size of advice, exploration time $\Theta\left(n^{2}\right)$ cannot be beaten, for some $n$-node graphs.

We will use the following construction from [10] of a family $\mathcal{H}_{X}$ of graphs.

Let $H$ be an $\frac{m}{2}$-regular graph with $m$ nodes, where $m$ is even, e.g., the complete bipartite graph. Let $T$ be the set of edges of any spanning tree of $H$. Let $S$ be the set of edges of $H$ outside $T$. Let $s=|S|=\frac{m^{2}}{4}-m+1$ and $S=\left\{e_{1}, e_{2}, \cdots, e_{s}\right\}$.

For $x \in\{0,1\}^{s} \backslash\left\{0^{s}\right\}$, the $(2 m)$-node graph $H_{x}$ is constructed from $H$ by taking two disjoint copies $H^{\prime}$ and $H^{\prime \prime}$ of $H$, and crossing some pairs of edges from one copy to the other. For $i=1, \cdots, s$, if the $i$-th bit of $x$ is 1 , then the edge $e_{i}=\left(u_{i}, v_{i}\right)$ is deleted from both copies of $H$ and two copies of $e_{i}$ are crossed between the two copies of $H$. More precisely, let $\left\{v_{1}, \cdots, v_{m}\right\}$ be the set of nodes of $H$ and let $v_{i}^{\prime}$ and $v_{i}^{\prime \prime}$ be the nodes corresponding to $v_{i}$, in $H^{\prime}$ and $H^{\prime \prime}$, respectively. Let $V^{\prime}$ and $V^{\prime \prime}$ be the sets of nodes of $H^{\prime}$ and $H^{\prime \prime}$, respectively. Define $H_{x}=\left(V^{\prime} \cup V^{\prime \prime}, E_{x}\right)$, where $E_{x}=\left\{\left(v_{i}^{\prime}, v_{j}^{\prime}\right),\left(v_{i}^{\prime \prime}, v_{j}^{\prime \prime}\right):\left(v_{i}, v_{j}\right) \in T\right\} \cup\left\{\left(v_{i}^{\prime}, v_{j}^{\prime \prime}\right),\left(v_{i}^{\prime \prime}, v_{j}^{\prime}\right)\right.$ : $e_{k}=\left(v_{i}, v_{j}\right) \in S$ and $\left.x_{k}=1\right\}$. Let $\mathcal{H}_{X}=\left\{H_{x}: x \in\{0,1\}^{s} \backslash\left\{0^{s}\right\}\right\}$.

According to the result from [10], for every node $v \in H$, there exists some sequence $x(v) \in\{0,1\}^{s} \backslash\left\{0^{s}\right\}$ such that if an exploration of $H$ performed according to some sequence $W$ of port numbers, starting from node $v_{1}$, visits node $v$ at most $s$ times, then in one of the copies $H^{\prime}$ or $H^{\prime \prime}$ in $H_{x(v)}$ the node $v^{\prime}$ or $v^{\prime \prime}$ is not visited at all, if the same sequence $W$ is used to explore the graph $H_{x(v)}$ starting from $v_{1}^{\prime}$. Intuitively, the result from [10] shows a class of graphs with the property that if some node in one of these graphs is not visited many times, then the exploration algorithm fails in some other graph of this class. There is no control in which graph of the class this will happen. We use the graphs from [10] as building blocks to prove a different kind of lower bound. Indeed, we construct a single graph having the property that if some of its nodes are not visited many times, then exploration must fail in this graph. This will prove a lower bound on exploration time for some graph, even if the agent knows the entire graph.

Using the graphs $H_{x} \in \mathcal{H}_{X}$ from [10] we construct the graph $\widehat{G}$ as follows. For any $1 \leq i \leq m$, let $v_{1}^{\prime}(i)$ be the node corresponding to node $v_{1}^{\prime}$ from $H^{\prime}$ in the graph $H_{x\left(v_{i}\right)}$. Connect the graphs $H_{x\left(v_{i}\right)}$, for $1 \leq i \leq m$, and an oriented cycle $C$ with nodes $\left\{y_{1}, \cdots, y_{m}\right\}$ (port numbers 0,1 are in clockwise order at each node of the cycle), by adding edges $\left(y_{i}, v_{1}^{\prime}(i)\right)$, for $1 \leq i \leq m$. The port numbers corresponding to these edges are: 2 at $y_{i}$ and $\frac{m}{2}$ at $v_{1}^{\prime}(i)$. The cycle $C$ is called the main cycle of $\widehat{G}$. See Fig. 1 .

Let $n=2 m^{2}+m$ be the number of nodes in $\widehat{G}$.

By the construction of $\widehat{G}$, any exploration algorithm with the agent starting from any node of the main cycle, has the following obliviousness property. For any step $i$ of the 


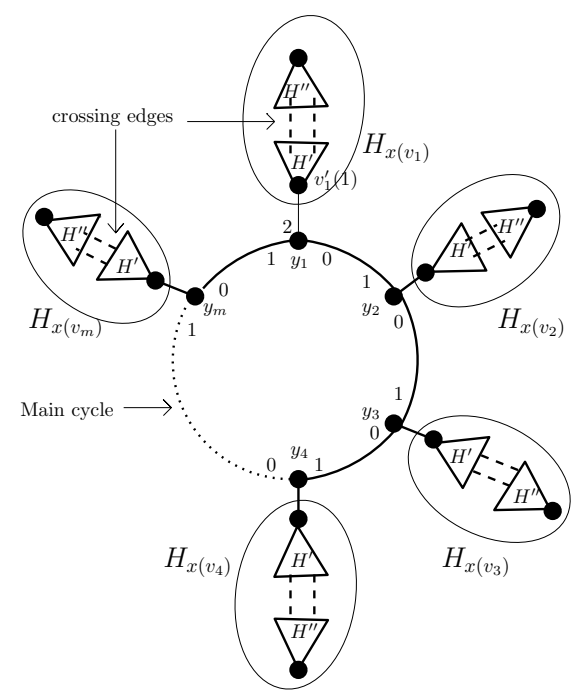

Figure 1 Construction of $\widehat{G}$.

algorithm, if the agent is at some node $v$ in this step, and the algorithm prescribes taking some port $p$ at this node, then the port $q$ through which the agent enters the adjacent node $w$ in the $(i+1)$-th step, and the degree of the node $w$ are predetermined (i.e., they are independent of the starting node in the main cycle). Intuitively, the agent does not learn anything during the algorithm execution. Therefore, every exploration algorithm with the agent starting from any node of the main cycle can be uniquely coded by a sequence of port numbers which the agent takes in consecutive steps of its exploration.

Let $\mathcal{A}$ be any exploration algorithm for $\widehat{G}$, and suppose that the agent starts from some node of the main cycle. We use · for concatenation of sequences.

- Lemma 6. Let $U$ be the sequence of port numbers corresponding to the movement of the agent according to algorithm $\mathcal{A}$, starting at some node of the main cycle $C$ of $\widehat{G}$. Then $U=B_{1}^{\prime} \cdot(2) \cdot B_{1} \cdot\left(\frac{m}{2}\right) \cdot B_{2}^{\prime} \cdot(2) \cdot B_{2} \cdot\left(\frac{m}{2}\right) \cdots B_{p}^{\prime} \cdot(2) \cdot B_{p} \cdot\left(\frac{m}{2}\right) \cdot B_{p+1}^{\prime}$, where each $B_{j}^{\prime}$ is a sequence of port numbers corresponding to the movement of the agent along $C$ and each $B_{j}$ is a sequence of port numbers corresponding to the movement of the agent inside some $H_{x\left(v_{i}\right)}$.

Call an exploration algorithm of $\widehat{G}$ non-repetitive, if the agent, starting from the main cycle, enters each $H_{x\left(v_{i}\right)}$, for $1 \leq i \leq m$, exactly once. By definition, the sequence of port numbers corresponding to a non-repetitive algorithm can be written as $D_{1}^{\prime} \cdot(2) \cdot D_{1} \cdot\left(\frac{m}{2}\right)$. $D_{2}^{\prime} \cdot(2) \cdot D_{2} \cdot\left(\frac{m}{2}\right) \cdots D_{m}^{\prime} \cdot(2) \cdot D_{m} \cdot\left(\frac{m}{2}\right) \cdot D_{m+1}^{\prime}$, where each $D_{j}^{\prime}$ is a sequence of port numbers corresponding to the movement of the agent along $C$ and each $D_{j}$ is a sequence of port numbers corresponding to the movement of the agent inside some $H_{x\left(v_{i}\right)}$. Notice that since the algorithm is non-repetitive, the number of blocks $D_{j}$ is exactly $m$.

The following lemma proves that in order to show a lower bound on the exploration time in $\widehat{G}$, it is enough to consider only the class of non-repetitive algorithms.

- Lemma 7. If the agent starts from some node of the main cycle of $\widehat{G}$ and executes any exploration algorithm $\mathcal{A}$ of $\widehat{G}$, then there exists a non-repetitive algorithm $\mathcal{A}^{\prime}$ for this agent, such that the exploration time of $\mathcal{A}^{\prime}$ is at most the exploration time of $\mathcal{A}$.

The next lemma implies that the sequence $U$ corresponding to a correct non-repetitive exploration algorithm must be long. 
- Lemma 8. Let $U=D_{1}^{\prime} \cdot(2) \cdot D_{1} \cdot\left(\frac{m}{2}\right) \cdot D_{2}^{\prime} \cdot(2) \cdot D_{2} \cdot\left(\frac{m}{2}\right) \cdots D_{m}^{\prime} \cdot(2) \cdot D_{m} \cdot\left(\frac{m}{2}\right) \cdot D_{m+1}^{\prime}$ be the sequence of port numbers corresponding to a non-repetitive algorithm. If there exists some $D_{i}$ such that the agent following $D_{i}$ in $H$ starting from node $v_{1}$ visits some node $v_{j}$ of $H$ at most $s$ times, then there exists a starting node in the main cycle of $\widehat{G}$, such that the agent starting at this node and following $U$ does not visit all the nodes of $\widehat{G}$.

- Theorem 9. Any exploration algorithm using any advice given by a map oracle must take time $\Omega\left(n^{2}\right)$ on graph $\widehat{G}$, for some starting node in the main cycle, for arbitrarily large $n$.

Proof. By Lemma 7, it is enough to consider only non-repetitive algorithms. Let $U=$ $D_{1}^{\prime} \cdot(2) \cdot D_{1} \cdot\left(\frac{m}{2}\right) \cdot D_{2}^{\prime} \cdot(2) \cdot D_{2} \cdot\left(\frac{m}{2}\right) \cdots D_{m}^{\prime} \cdot(2) \cdot D_{m} \cdot\left(\frac{m}{2}\right) \cdot D_{m+1}^{\prime}$ be the sequence of port numbers corresponding to such an algorithm. Then by Lemma 8 , for each $i, 1 \leq i \leq m$, the agent following $D_{i}$ in $H$ starting from node $v_{1}$ visits each node $v_{j}$ of $H$, for $1 \leq j \leq m$, at least $s+1$ times. Therefore the length of $D_{i}$ is at least $(s+1) m$. Hence, the length of $U$ is at least $\sum_{i=1}^{m}(s+1) m=(s+1) m^{2}=m^{2}\left(\frac{m^{2}}{4}-m+1\right)$. Since $n=2 m^{2}+m$, the length of $U$ is in $\Omega\left(n^{2}\right)$.

Theorem 9 shows that, for some $n$-node graph, no advice given by a map oracle can help to explore this graph in time better than $\Theta\left(n^{2}\right)$. It is then natural to ask what is the minimum size of advice to achieve time $\Theta\left(n^{2}\right)$ with a map oracle, for every $n$-node graph. Our next result shows that any exploration algorithm using advice of size $n^{\delta}$ for $\delta<\frac{1}{3}$, must take time $\omega\left(n^{2}\right)$, on some $n$-node graph.

Fix a constant $\epsilon<\frac{1}{2}$. Let $H$ be an $\frac{m}{2}$-regular graph with $m$ nodes, where $m$ is even. Let $\left\{v_{1}, \cdots, v_{m}\right\}$ be the set of nodes of $H$. Consider a subset $Z \subset\{1,2, \cdots, m\}$ of size $m^{\epsilon}$. Let $p=m^{\epsilon}$ and $n=2 m p+p$. We construct an $n$-node graph $\widehat{G_{Z}}$ from $H$. The construction of $\widehat{G_{Z}}$ is similar to the construction of $\widehat{G}$ at the beginning of this section. Let $Z=\left\{z_{1}, z_{2}, \cdots, z_{p}\right\}$. To construct $\widehat{G_{Z}}$, connect the (previously described) graphs $H_{x\left(v_{z_{i}}\right)}$, for $1 \leq i \leq p$, and an oriented cycle $C^{\prime}$ (called the main cycle) with nodes $\left\{y_{1}, \cdots, y_{p}\right\}$, by adding edges $\left(y_{i}, v_{1}^{\prime}\left(z_{i}\right)\right)$, for $1 \leq i \leq p$. The port numbers corresponding to these edges are: 2 at $y_{i}$ and $\frac{m}{2}$ at $v_{1}^{\prime}\left(z_{i}\right)$. Note that the same obliviousness property applies to exploration algorithms in graphs $\widehat{G_{Z}}$, when the agent starts from a node of the main cycle.

Let $\widehat{\mathcal{G}_{Z}}$ be the set of all possible graphs $\widehat{G_{Z}}$ constructed from $H$. We have $\left|\widehat{\mathcal{G}_{Z}}\right|=\left(\begin{array}{c}m \\ p\end{array}\right)$.

- Theorem 10. For any $\epsilon<\frac{1}{2}$, any exploration algorithm using advice of size o( $\left.n^{\frac{\epsilon}{1+\epsilon}} \log n\right)$ must take time $\omega\left(n^{2}\right)$ on some graph of the class $\widehat{\mathcal{G}_{Z}}$ and for some starting node in the main cycle of this graph, for arbitrarily large $n$.

Proof. Since $\epsilon<\frac{1}{2}$, there exists an integer $c$ such that $\epsilon<\frac{c-1}{2 c-1}$. We show that if the size of the advice is at most $\frac{1}{2 c} m^{\epsilon} \log \left(m^{1-\epsilon}\right) \leq \frac{1-\epsilon}{2 c(1+\epsilon)}\left(\frac{n}{2}\right)^{\frac{\epsilon}{1+\epsilon}} \log \frac{n}{2}$, then there exists a graph in $\widehat{\mathcal{G}_{Z}}$ for which the time required for exploration is $\omega\left(n^{2}\right)$. We have $\left|\widehat{\mathcal{G}_{Z}}\right|=\left(\begin{array}{c}m \\ m^{\epsilon}\end{array}\right) \geq\left(m^{1-\epsilon}\right)^{m^{\epsilon}}$. There are fewer than $\left(m^{1-\epsilon}\right)^{\frac{m^{\epsilon}}{c}}$ different binary strings of length at most $\frac{1}{2 c} m^{\epsilon} \log \left(m^{1-\epsilon}\right)$. By the pigeonhole principle, there exists a family of graphs $\widehat{\mathcal{G}} \subset \widehat{\mathcal{G} Z}$ of size at least $\left(m^{1-\epsilon}\right)^{(c-1) \frac{m^{\epsilon}}{c}}$ such that all the graphs in $\widehat{\mathcal{G}}$ get the same advice.

Define $F(\widehat{\mathcal{G}})=\bigcup\left\{\left\{v_{z_{1}}, v_{z_{2}}, \cdots, v_{z_{p}}\right\}: Z=\left\{z_{1}, z_{2}, \cdots, z_{p}\right\}\right.$ and $\left.\widehat{G_{Z}} \in \widehat{\mathcal{G}}\right\}$. Intuitively, $F(\widehat{\mathcal{G}})$ is the subset of nodes of $H$, such that for each $v \in F(\widehat{\mathcal{G}})$, there exists some graph in $\widehat{\mathcal{G}}$ that contains $H_{x(v)}$ as a subgraph. 
Claim: $|F(\widehat{\mathcal{G}})| \geq|\widehat{\mathcal{G}}|^{\frac{1}{p}}$.

Proof. We prove the claim by contradiction. Suppose that $|F(\widehat{\mathcal{G}})|<|\widehat{\mathcal{G}}|^{\frac{1}{p}}$. Each graph in $\widehat{\mathcal{G}}$ has $p$ different subgraphs $H_{x(v)}$, where $v \in|F(\widehat{\mathcal{G}})|$. There are $\left(\begin{array}{c}|F(\widehat{\mathcal{G}})| \\ p\end{array}\right)$ different graphs in $\widehat{\mathcal{G}}$ which is at most $|F(\widehat{\mathcal{G}})|^{p}<|\widehat{\mathcal{G}}|$. This contradiction proves the claim.

Consider the exploration of some graph $\widehat{G_{Z}} \in \widehat{\mathcal{G}}$ starting from the main cycle. Let $U=D_{1}^{\prime} \cdot(2) \cdot D_{1} \cdot\left(\frac{m}{2}\right) \cdot D_{2}^{\prime} \cdot(2) \cdot D_{2} \cdot\left(\frac{m}{2}\right) \cdots D_{p}^{\prime} \cdot(2) \cdot D_{m} \cdot\left(\frac{m}{2}\right) \cdot D_{p+1}^{\prime}$ be the sequence of port numbers corresponding to a non-repetitive algorithm exploring $\widehat{G_{Z}}$. Then for each $i, 1 \leq i \leq p$, the agent following $D_{i}$ in $H$ starting from node $v_{1}$ must visit each node $v \in F(\widehat{\mathcal{G}})$ at least $s+1$ times. (Otherwise, there would exist a graph in $\widehat{\mathcal{G}}$ and a starting node in the main cycle, for which one node would not be explored by $U$ ). Hence, for sufficiently large $m$, the length of $D_{i}$ is at least $(s+1)|F(\widehat{\mathcal{G}})| \geq \frac{m^{2}}{5} m^{\frac{(c-1)(1-\epsilon)}{c}}$, because $s \geq \frac{m^{2}}{5}$. Therefore, the length of $U$ is at least $p \frac{m^{2}}{5} m^{\frac{(c-1)(1-\epsilon)}{c}}=\frac{1}{5} m^{\epsilon} m^{2} m^{\frac{(c-1)(1-\epsilon)}{c}}=\frac{1}{5} m^{2+\epsilon+\frac{(c-1)(1-\epsilon)}{c}}=\frac{1}{5} m^{2+2 \epsilon+\left(\frac{c-1}{c}+\frac{1-2 c}{c} \epsilon\right)}$. Since $\epsilon<\frac{c-1}{2 c-1}$, we have $\left(\frac{c-1}{c}+\frac{1-2 c}{c} \epsilon\right)>0$. Therefore, the length of $U$ is in $\omega\left(\left(2 m^{1+\epsilon}+m^{\epsilon}\right)^{2}\right)=$ $\omega\left(n^{2}\right)$, and hence exploration time is in $\omega\left(n^{2}\right)$.

Since $\epsilon<\frac{1}{2}$ implies $\frac{\epsilon}{1+\epsilon}<\frac{1}{3}$, Theorem 10 yields the following corollary.

- Corollary 11. For any $\delta<\frac{1}{3}$, any exploration algorithm using advice of size o( $\left.n^{\delta}\right)$ must take time $\omega\left(n^{2}\right)$ on some $n$-node graph, for arbitrarily large $n$.

\subsection{Instance oracle}

For the instance oracle we show a general lower bound on the size of advice needed to achieve a given exploration time. The main corollaries of this lower bound are:

- the size of advice $\Theta(n \log n)$ from Proposition 5, sufficient to achieve linear exploration time, cannot be beaten;

- for advice of linear size, exploration time must be quadratic.

To prove our lower bound we will use the following construction.

Let $G$ be an $\frac{n}{4}$-regular $\frac{n}{2}$-node graph, where $n$ is divisible by 4 . We can use, for example the complete bipartite graph with $\frac{n}{2}$ nodes. Let $m=\frac{n}{2}$. Let $v_{1}, v_{2}, \cdots, v_{m}$ be the nodes of $G$. Let $x=\left(x_{1}, x_{2}, \cdots, x_{m}\right)$ be a sequence of $m$ integers where $0 \leq x_{i} \leq \frac{m}{2}-1$, for $i=1, \cdots, m$. Let $X$ be the set of all such sequences.

We construct an $n$-node graph $G_{x}$ as follows. For each $i=1, \cdots, m$, add a new node $v_{i}^{\prime}$ of degree 1 to $G$. Replace the port number $x_{i}$ at $v_{i}$ by port number $\frac{m}{2}$. Add the edge $\left(v_{i}, v_{i}^{\prime}\right)$ with the port number $x_{i}$ at $v_{i}$. An example of the construction of $G_{x}$ from $G$ is shown in Fig. 2. Let $\mathcal{G}_{X}$ be the set of all possible graphs $G_{x}$ constructed from $G$.

- Theorem 12. For any function $\phi: \mathbb{N} \longrightarrow \mathbb{N}$, and for any exploration algorithm using advice of size o $(n \phi(n))$, this algorithm must take time $\Omega\left(\frac{n^{2}}{2^{\phi(n)}}\right)$ on some $n$-node graph from the family $\mathcal{G}_{X}$, for arbitrarily large integers $n$.

Proof. Let $n$ be divisible by 4 . We show that if the size of the advice is at most $\frac{n \phi(n)}{4}-1$, then there exists an $n$-node graph in the family $\mathcal{G}_{X}$, for which the time required for exploration is $\Omega\left(\frac{n^{2}}{2^{\phi(n)}}\right)$. We have $\left|\mathcal{G}_{X}\right|=|X|=\left(\frac{m}{2}\right)^{m}$. There are fewer than $2^{\frac{m \phi(2 m)}{2}}=\left(2^{\phi(2 m)}\right)^{\frac{m}{2}}$ different binary strings of length at most $\frac{2 m \phi(2 m)}{4}-1=\frac{n \phi(n)}{4}-1$. By the pigeonhole principle, there exists a family of graphs $\mathcal{G} \subset \mathcal{G}_{X}$, of size at least $\frac{\left(\frac{m}{2}\right)^{m}}{\left(2^{\phi(2 m)}\right)^{\frac{m}{2}}}$, such that all the graphs in $\mathcal{G}$ get the same advice. Let $Y=\left\{x \in X: G_{x} \in \mathcal{G}\right\}$. Let $z=\frac{m}{2^{\phi(2 m)+2}}$ and let 


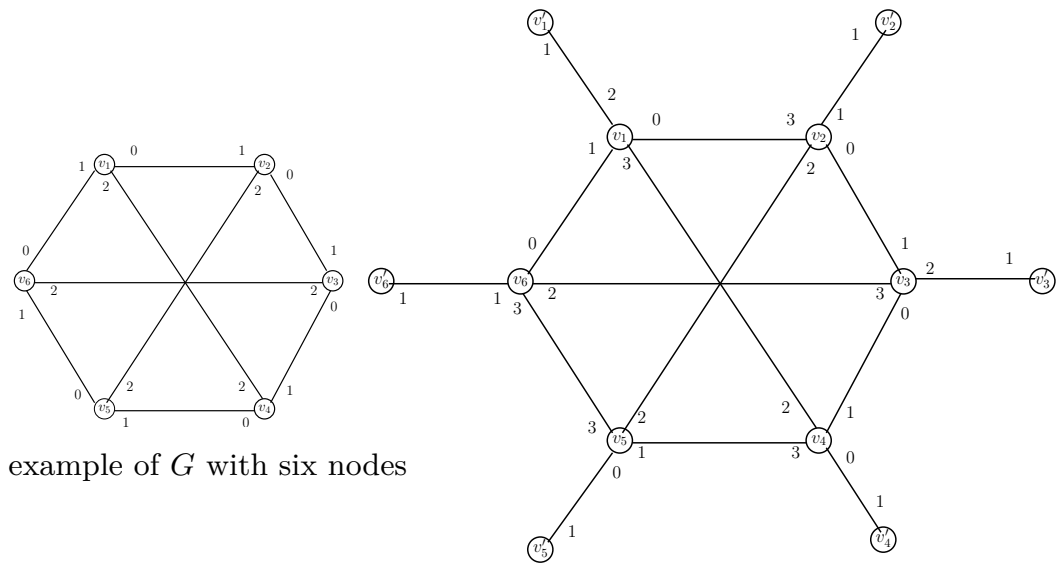

(b) $G_{x}$ for the sequence $x=(2,1,2,0,0,1)$

Figure 2 The construction of $G_{x}$ from $G$.

$J=\left\{j:\left|\left\{x_{j}: x \in Y\right\}\right| \geq z\right\}$. Intuitively, $J$ is the set of indices, for which the set of terms of sequences $x$ that produce graphs from $\mathcal{G}$ is large. Let $p=|J|$.

Claim: $\quad p>\frac{m}{2}$.

Proof. We prove the claim by contradiction. Suppose that $p \leq \frac{m}{2}$. Since $p \leq \frac{m}{2}$ and $z<\frac{m}{2}$, we have $\left(\frac{m}{2}\right)^{p} \cdot z^{m-p} \leq\left(\frac{m}{2}\right)^{\frac{m}{2}} \cdot z^{\frac{m}{2}}$. Note that for all $i \in\{1,2, \cdots, m\} \backslash J$, we have $\left|\left\{x_{i}: x \in Y\right\}\right|<z$, and for $j \in J$, we have $\left|\left\{x_{j}: x \in Y\right\}\right| \leq \frac{m}{2}$. Therefore, $|\mathcal{G}|<\left(\frac{m}{2}\right)^{p} \cdot z^{m-p}$. Hence, $|\mathcal{G}|<\left(\frac{m}{2}\right)^{\frac{m}{2}}\left(\frac{m}{2^{\phi(2 m)+2}}\right)^{\frac{m}{2}}=\frac{m^{m}}{2^{\frac{m}{2}}\left(2^{\phi(2 m)+2}\right)^{\frac{m}{2}}}<|\mathcal{G}|$, which is a contradiction. This proves the claim.

Consider any exploration algorithm for the class $\mathcal{G}$. There exists a graph $G_{x} \in \mathcal{G}$, such that, at each node $v_{j}$ of $G_{x}$, for $j \in J$, the agent must take all the ports in $\left\{x_{j}: x \in Y\right\}$. Indeed, suppose that the agent does not take some port $x_{j}$, where $j \in J$ and $x \in Y$. Consider the exploration of any graph $G_{x^{\prime}} \in \mathcal{G}$, where $x_{j}^{\prime}=x_{j}$. Since the agent can visit $v_{j}^{\prime}$ only coming from $v_{j}$, using port $x_{j}^{\prime}$ in $G_{x^{\prime}}$, the node $v_{j}^{\prime}$ remains unexplored, as the port $x_{j}^{\prime}$ at $v_{j}$ is never used, which is a contradiction. Hence, the agent must visit at least $\frac{m}{2^{\phi(2 m)+2}}$ ports at each node $v_{j}$ for $j \in J$. Since $|J|>\frac{m}{2}$, the time required for exploration is at least $\frac{m^{2}}{2^{\phi(2 m)+3}}$, i.e., it is at least $\frac{n^{2}}{2^{\phi(n)+5}}$.

If $\phi(n)=c$ where $c$ is a constant, then Theorem 12 implies that any exploration algorithm using advice of size at most $\frac{c n}{2}$, must take time at least $\frac{n^{2}}{2^{c+3}}$. This implies that, if the size of advice is at most $c^{\prime} n$, for any constant $c^{\prime}$, then exploration time is $\Omega\left(n^{2}\right)$. Hence we have the following corollary.

- Corollary 13. Any exploration algorithm using advice of size $O(n)$ must take time $\Omega\left(n^{2}\right)$ on some $n$-node graph, for arbitrarily large $n$.

For $\phi(n) \in o(\log n)$, Theorem 12 implies an exploration time $\omega(n)$ which shows that the upper bound on the size of advice from Proposition 5 is asymptotically tight for exploration in linear time. The following corollary improves this statement significantly, showing that exploration time is very sensitive to the size of advice at the threshold $\Theta(n \log n)$ of the latter. 
- Corollary 14. Consider any constant $\epsilon<2$. Any exploration algorithm using advice of size o $(n \log n)$ must take time $\Omega\left(n^{\epsilon}\right)$, on some $n$-node graph, for arbitrarily large $n$.

Proof. If the size of advice is $o(n \log n)$, then it is $n \phi(n)$, where $\phi(n)=\frac{\log n}{f(n)}$, with $f(n) \rightarrow \infty$ as $n \rightarrow \infty$. Theorem 12 implies that exploration time must be $\Omega\left(\frac{n^{2}}{2^{\frac{\log n}{f(n)}}}\right)=\Omega\left(\frac{n^{2}}{n^{\frac{1}{f(n)}}}\right)$. Since, for any constant $\delta>0$, we have $n^{\frac{1}{f(n)}} \in O\left(n^{\delta}\right)$, the corollary holds.

\section{Exploration of Hamiltonian graphs}

In this section we turn attention to Hamiltonian graphs. These graphs have a special feature from the point of view of exploration: with sufficiently large advice of appropriate type, the agent can explore a Hamiltonian graph without any loss of time, visiting each node exactly once, i.e., in time $n-1$, for $n$-node graphs. Indeed, an instance oracle can give as advice the sequence of port numbers along a Hamiltonian cycle, from the starting node of the agent, and then the agent takes the prescribed ports in $n-1$ consecutive steps. Since it is enough to give $n-1$ port numbers, and the binary representation of each port number uses $O(\log n)$ bits, advice of size $O(n \log n)$, given by an instance oracle, suffices.

We show that neither the quality nor the size of advice can be decreased to achieve the goal of optimal exploration of Hamiltonian graphs. To prove the first statement, we show a graph which is impossible to explore in time $n-1$ when advice of any size is given by a map oracle. Indeed, we construct an $n$-node Hamiltonian graph for which even knowing the entire map of the graph (but not knowing its starting node) an agent must use time $\Omega\left(n^{2}\right)$ to explore it. To prove the second statement, we construct a class of $n$-node Hamiltonian graphs for which advice of size $o(n \log n)$, even given by an instance oracle, is not enough to permit exploration of graphs in this class in time $n-1$. Indeed, we show more: any exploration algorithm using such advice must exceed the optimal time $n-1$ by a summand $n^{\epsilon}$, for any $\epsilon<1$, on some graph of this class.

In order to prove the first result, we construct a $(3 n)$-node Hamiltonian graph $\tilde{G}$ from the $n$-node graph $\widehat{G}$ described in Section 3.1. First, we consider an $\frac{m}{2}$-regular $m$-node Hamiltonian graph $H$ (for example, the complete bipartite graph). Let $v_{1}, v_{2}, \cdots, v_{m}$ be the nodes of $H$ along a Hamiltonian cycle. The graph $\widehat{G}$ is constructed from $H$ as described in Section 3.1, where the Hamiltonian path $\left(v_{1}, v_{2}, \cdots, v_{m}\right)$ is taken as the spanning tree $T$. We construct the Hamiltonian graph $\tilde{G}$ from the graph $\widehat{G}$ as follows. Denote by $d(v)$ the degree of node $v$ in $\widehat{G}$. For each node $v$ in $\widehat{G}$, consider a cycle of three nodes $v(1), v(2)$, and $v(3)$, in $\tilde{G}$, with port numbers $3 d(v), 3 d(v)+1$ in clockwise order at each of these three nodes. For each edge $(u, v)$ in $\widehat{G}$, such that the port numbers corresponding to this edge are $p$ at $u$ and $q$ at $v$, add, in $\tilde{G}$, the edges $(u(i), v(j))$, for $1 \leq i, j \leq 3$, with the following port numbers. The port numbers corresponding to edge $(u(i), v(j))$ are: $p+(j-1) d(u)$ at $u(i)$ and $q+(i-1) d(v)$ at $v(j)$, see Fig. 3 .

- Lemma 15. The graph $\tilde{G}$ is Hamiltonian.

Let $\mathcal{A}$ be an exploration algorithm for $\tilde{G}$ starting from node $y_{i}(1)$, for some $i \leq m$. We describe the following algorithm $\mathcal{A}^{*}$ on $\widehat{G}$, starting from node $y_{i}$. Ignore all moves of $\mathcal{A}$ taking port $3 d(v)$ or $3 d(v)+1$ at a node $v(j)$, for $1 \leq j \leq 3$, of $\tilde{G}$. Replace every move of $\mathcal{A}$ taking port $r=p+(i-1) d(v)$, at node $v(j)$, for $1 \leq j \leq 3$, in $\tilde{G}$, where $0 \leq p \leq d(v)-1$, by a move taking port $p$ in $\widehat{G}$.

Then the agent executing $\mathcal{A}^{*}$ in $\widehat{G}$, starting from the main cycle, explores all the nodes. The time used by $\mathcal{A}^{*}$ in $\widehat{G}$ does not exceed the time used by $\mathcal{A}$ in $\tilde{G}$. Since, by Theorem 9 , any 

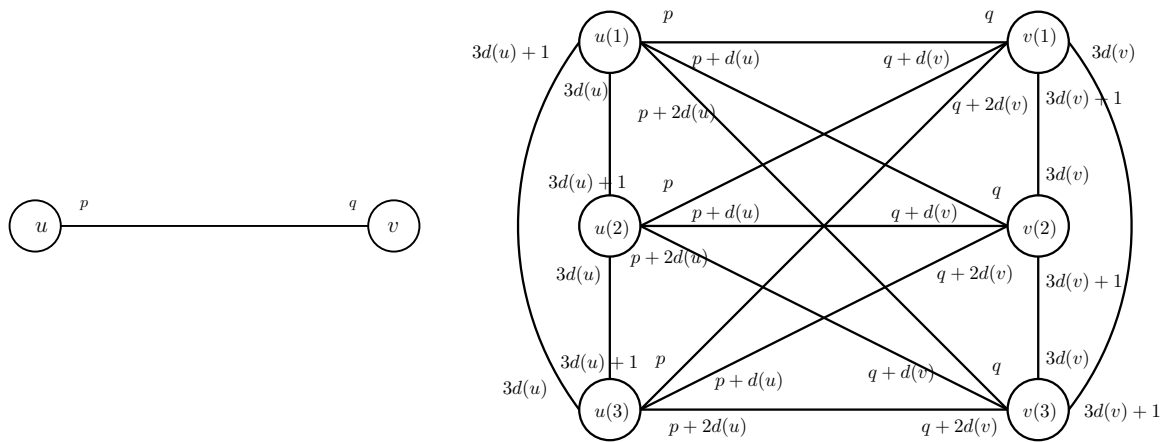

Figure 3 The construction of $\tilde{G}$ from $\widehat{G}$.

exploration algorithm for $\widehat{G}$, starting from the main cycle, must take time $\Omega\left(n^{2}\right)$, algorithm $\mathcal{A}$ must take time $\Omega\left(n^{2}\right)$ to explore $\tilde{G}$. Replacing $3 n$ by $n$ we have the following theorem.

- Theorem 16. Any exploration algorithm using any advice given by a map oracle must take time $\Omega\left(n^{2}\right)$ on some $n$-node Hamiltonian graph, for arbitrarily large $n$.

Our last result shows that advice of size $o(n \log n)$ causes significant increase of exploration time for some Hamiltonian graphs, as compared to optimal time $n-1$ achievable with advice of size $O(n \log n)$, given by an instance oracle.

- Theorem 17. For any constant $\epsilon<1$, and for any exploration algorithm using advice of size $o(n \log n)$, this algorithm must take time $n+n^{\epsilon}$, on some $n$-node Hamiltonian graph, for arbitrarily large $n$.

\section{Conclusion}

Most of our lower bounds on the size of advice are either exactly or asymptotically tight. The lower bound $\log \log \log n-\Theta(1)$ on the size of advice sufficient to explore all $n$-node graphs in polynomial time is exactly tight: with advice of any such size, polynomial exploration is possible, and with advice of any smaller size it is not. For an instance oracle, the lower bound $\Omega(n \log n)$ on the size of advice sufficient to explore $n$-node graphs in $O(n)$ time is asymptotically tight, as we gave a linear time exploration algorithm using advice of size $O(n \log n)$. An exception to this tightness is the lower bound on the size of advice given by a map oracle, permitting exploration in time $O\left(n^{2}\right)$. While the natural upper bound is $O(n \log n)$, our lower bound is only $n^{\delta}$ for any $\delta<\frac{1}{3}$. Hence the main remaining question is:

What is the smallest advice, given by a map oracle, permitting exploration of $n$-node graphs in time $O\left(n^{2}\right)$ ?

Acknowledgements. We are grateful to Adrian Kosowski for early discussions on the subject of this paper and for drawing our attention to [10].

\section{References}

1 Serge Abiteboul, Stephen Alstrup, Haim Kaplan, Tova Milo, and Theis Rauhe. Compact labeling scheme for ancestor queries. SIAM J. Comput., 35(6):1295-1309, 2006. doi: 10.1137/S0097539703437211.

2 Susanne Albers and Monika Rauch Henzinger. Exploring unknown environments. SIAM J. Comput., 29(4):1164-1188, 2000. doi:10.1137/S009753979732428X. 
3 Romas Aleliunas, Richard M. Karp, Richard J. Lipton, László Lovász, and Charles Rackoff. Random walks, universal traversal sequences, and the complexity of maze problems. In 20th Annual Symposium on Foundations of Computer Science, San Juan, Puerto Rico, 29-31 October 1979, pages 218-223. IEEE Computer Society, 1979. doi:10.1109/SFCS.1979.34.

4 Baruch Awerbuch, Margrit Betke, Ronald L. Rivest, and Mona Singh. Piecemeal graph exploration by a mobile robot. Inf. Comput., 152(2):155-172, 1999. doi:10.1006/inco. 1999. 2795.

5 Eldad Bar-Eli, Piotr Berman, Amos Fiat, and Peiyuan Yan. Online navigation in a room. J. Algorithms, 17(3):319-341, 1994. doi:10.1006/jagm.1994.1039.

6 Michael A. Bender, Antonio Fernández, Dana Ron, Amit Sahai, and Salil P. Vadhan. The power of a pebble: Exploring and mapping directed graphs. Inf. Comput., 176(1):1-21, 2002. doi:10.1006/inco.2001.3081.

7 Michael A. Bender and Donna K. Slonim. The power of team exploration: Two robots can learn unlabeled directed graphs. In 35th Annual Symposium on Foundations of Computer Science, Santa Fe, New Mexico, USA, 20-22 November 1994, pages 75-85. IEEE Computer Society, 1994. doi:10.1109/SFCS.1994.365703.

8 Margrit Betke, Ronald L. Rivest, and Mona Singh. Piecemeal learning of an unknown environment. Machine Learning, 18(2-3):231-254, 1995. doi:10.1007/BF00993411.

9 Avrim Blum, Prabhakar Raghavan, and Baruch Schieber. Navigating in unfamiliar geometric terrain. SIAM J. Comput., 26(1):110-137, 1997. doi:10.1137/S0097539791194931.

10 Allan Borodin, Walter L. Ruzzo, and Martin Tompa. Lower bounds on the length of universal traversal sequences. J. Comput. Syst. Sci., 45(2):180-203, 1992. doi:10.1016/ 0022-0000 (92) 90046-L.

11 Jérémie Chalopin, Shantanu Das, and Adrian Kosowski. Constructing a map of an anonymous graph: Applications of universal sequences. In Chenyang Lu, Toshimitsu Masuzawa, and Mohamed Mosbah, editors, Principles of Distributed Systems - 14th International Conference, OPODIS 2010, Tozeur, Tunisia, December 14-17, 2010. Proceedings, volume 6490 of Lecture Notes in Computer Science, pages 119-134. Springer, 2010. doi : 10.1007/978-3-642-17653-1_10.

12 Xiaotie Deng, Tiko Kameda, and Christos H. Papadimitriou. How to learn an unknown environment I: the rectilinear case. J. ACM, 45(2):215-245, 1998. doi:10.1145/274787. 274788

13 Dariusz Dereniowski and Andrzej Pelc. Drawing maps with advice. J. Parallel Distrib. Comput., 72(2):132-143, 2012. doi:10.1016/j.jpdc.2011.10.004.

14 Krzysztof Diks, Pierre Fraigniaud, Evangelos Kranakis, and Andrzej Pelc. Tree exploration with little memory. J. Algorithms, 51(1):38-63, 2004. doi:10.1016/j.jalgor.2003.10. 002 .

15 Stefan Dobrev, Rastislav Královic, and Euripides Markou. Online graph exploration with advice. In Guy Even and Magnús M. Halldórsson, editors, Structural Information and Communication Complexity - 19th International Colloquium, SIROCCO 2012, Reykjavik, Iceland, June 30-July 2, 2012, Revised Selected Papers, volume 7355 of Lecture Notes in Computer Science, pages 267-278. Springer, 2012. doi:10.1007/978-3-642-31104-8_23.

16 Christian A. Duncan, Stephen G. Kobourov, and V. S. Anil Kumar. Optimal constrained graph exploration. ACM Trans. Algorithms, 2(3):380-402, 2006. doi:10.1145/1159892. 1159897.

17 Yuval Emek, Pierre Fraigniaud, Amos Korman, and Adi Rosén. Online computation with advice. Theor. Comput. Sci., 412(24):2642-2656, 2011. doi:10.1016/j.tcs.2010.08.007.

18 Pierre Fraigniaud, Cyril Gavoille, David Ilcinkas, and Andrzej Pelc. Distributed computing with advice: information sensitivity of graph coloring. Distributed Computing, 21(6):395403, 2009. doi:10.1007/s00446-008-0076-y. 
19 Pierre Fraigniaud and David Ilcinkas. Digraphs exploration with little memory. In Volker Diekert and Michel Habib, editors, STACS 2004, 21st Annual Symposium on Theoretical Aspects of Computer Science, Montpellier, France, March 25-27, 2004, Proceedings, volume 2996 of Lecture Notes in Computer Science, pages 246-257. Springer, 2004. doi:10.1007/ 978-3-540-24749-4_22.

20 Pierre Fraigniaud, David Ilcinkas, and Andrzej Pelc. Tree exploration with advice. Inf. Comput., 206(11):1276-1287, 2008. doi:10.1016/j.ic.2008.07.005.

21 Pierre Fraigniaud, David Ilcinkas, and Andrzej Pelc. Communication algorithms with advice. J. Comput. Syst. Sci., 76(3-4):222-232, 2010. doi:10.1016/j.jcss.2009.07.002.

22 Pierre Fraigniaud, Amos Korman, and Emmanuelle Lebhar. Local MST computation with short advice. Theory Comput. Syst., 47(4):920-933, 2010. doi:10.1007/ s00224-010-9280-9.

23 Emanuele G. Fusco and Andrzej Pelc. Trade-offs between the size of advice and broadcasting time in trees. Algorithmica, 60(4):719-734, 2011. doi:10.1007/s00453-009-9361-9.

24 Emanuele G. Fusco, Andrzej Pelc, and Rossella Petreschi. Topology recognition with advice. Inf. Comput., 247:254-265, 2016. doi:10.1016/j.ic.2016.01.005.

25 Cyril Gavoille, David Peleg, Stéphane Pérennes, and Ran Raz. Distance labeling in graphs. J. Algorithms, 53(1):85-112, 2004. doi:10.1016/j.jalgor.2004.05.002.

26 David Ilcinkas, Dariusz R. Kowalski, and Andrzej Pelc. Fast radio broadcasting with advice. Theor. Comput. Sci., 411(14-15):1544-1557, 2010. doi:10.1016/j.tcs.2010.01.004.

27 Amos Korman, Shay Kutten, and David Peleg. Proof labeling schemes. Distributed Computing, 22(4):215-233, 2010. doi:10.1007/s00446-010-0095-3.

28 Robert Krauthgamer, editor. Proceedings of the Twenty-Seventh Annual ACM-SIAM Symposium on Discrete Algorithms, SODA 2016, Arlington, VA, USA, January 10-12, 2016. SIAM, 2016. doi:10.1137/1.9781611974331.

29 Nicolas Nisse and David Soguet. Graph searching with advice. Theor. Comput. Sci., 410(14):1307-1318, 2009. doi:10.1016/j.tcs.2008.08.020.

30 Petrisor Panaite and Andrzej Pelc. Exploring unknown undirected graphs. J. Algorithms, 33(2):281-295, 1999. doi:10.1006/jagm.1999.1043.

31 Petrisor Panaite and Andrzej Pelc. Optimal broadcasting in faulty trees. J. Parallel Distrib. Comput., 60(5):566-584, 2000. doi:10.1006/jpdc.2000.1625.

32 Andrzej Pelc and Anas Tiane. Efficient grid exploration with a stationary token. Int. J. Found. Comput. Sci., 25(3):247-262, 2014. doi:10.1142/S0129054114500129.

33 Nageswara S. V. Rao, Srikumar Kareti, Weimin Shi, and S. Sitharama Iyengar. Robot navigation in unknown terrains: Introductory survey of non-heuristic algorithms, Jul 1993. doi: $10.2172 / 10180101$.

34 Omer Reingold. Undirected connectivity in log-space. J. ACM, 55(4):17:1-17:24, 2008. doi : 10.1145/1391289.1391291.

35 A. Pelc Y. Dieudonné. Impact of knowledge on election time in anonymous networks. In Proc. 29th ACM Symposium on Parallelism in Algorithms and Architectures (SPAA 2017), 2017. doi:10.1007/978-3-642-17653-1_10. 